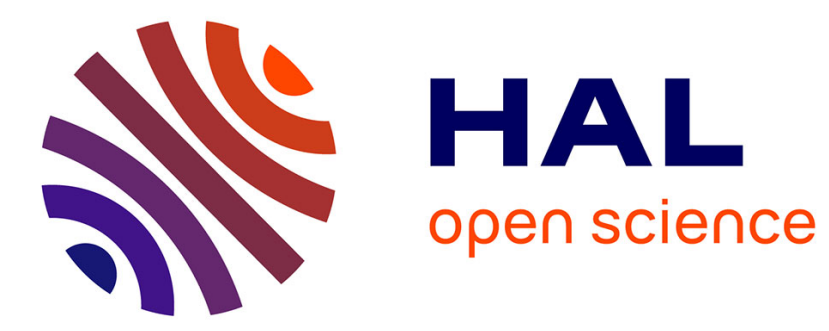

\title{
La forêt et un parc naturel régional : Normandie-Maine
}

Roland Perrier

\section{To cite this version:}

Roland Perrier. La forêt et un parc naturel régional: Normandie-Maine. Revue forestière française, 1970, 22 (S), pp.783-791. 10.4267/2042/20468 . hal-03386286

\section{HAL Id: hal-03386286 \\ https://hal.science/hal-03386286}

Submitted on 19 Oct 2021

HAL is a multi-disciplinary open access archive for the deposit and dissemination of scientific research documents, whether they are published or not. The documents may come from teaching and research institutions in France or abroad, or from public or private research centers.
L'archive ouverte pluridisciplinaire HAL, est destinée au dépôt et à la diffusion de documents scientifiques de niveau recherche, publiés ou non, émanant des établissements d'enseignement et de recherche français ou étrangers, des laboratoires publics ou privés. 


\title{
LA FORÊT ET UN PARC NATUREL RÉGIONAL : NORMANDIE - MAINE
}

\author{
R. PERRIER
}

Class. Oxford $681: 907.11(44)$

" Le territoire de tout ou partie d'une ou de plusieurs communes peut être classé en " parc naturel régional "Iorsqu'il présente un intérêt particulier, par la qualité de son patrimoine naturel et culturel, pour la détente, le repos des hommes et le tourisme, et qu'il importe de le protéger et de l'organiser. "

Tel est l'article 1 du décret $n^{\circ}$ 67-158 du $1^{\text {er }}$ mars 1967, instituant les parcs naturels régionaux.

\section{GENESE DU PROJET DE PARC NATUREL REGIONAL NORMANDIE-MAINE}

Dès lors, il n'est pas surprenant que, dans sa séance du 9 janvier 1968, le Conseil général du département de l'Orne ait pris l'initiative de demander le classement en parc naturel régional d'une zone s'appuyant sur les massifs forestiers d'Ecouves et des Andaines : Ecouves, vaste ensemble de près de 15.000 ha, dont plus de 8.000 ha propriété domaniale, aux portes mêmes d'Alençon; Andaines, plus à l'Est, enchâssant la station thermale de Bagnoles de l'Orne; entre ces deux môles, un bocage où l'agriculture est en perte de vitesse avec, au centre, le magnifique château de Carrouges. Région par conséquent attachante, particulièrement propice à "la détente et au repos des hommes", et à laquelle sa relative proximité de l'agglomération parisienne et sa situation privilégiée sur l'axe Paris - Nord Bretagne, confèrent de sérieux atouts sur le plan touristique. II n'est pas surprenant non plus que, dans un deuxième temps, la Commission interministérielle des Parcs naturels régionaux se soit prononcée, le 21 juin 1968, en faveur de la " prise en considération " du projet, tout en demandant un examen plus attentif de l'aire à assigner à l'ètude de reconnaissance, pour que soient éventuellement incorporés les secteurs de la forêt de Perseigne et des Alpes Mancelles, situés aux environs immédiats d'Alençon. Finalement, le périmètre retenu devait englober une surface totale de l'ordre de 260.000 hectares, comprenant les adjonctions précitées, et incluant par surc:oît la forêt domaniale de Sillé-le-Guillaume, où la présence d'un lac de 35 ha avait d'ores et déjà suscifé l'aménagement d'une base de loisirs, ainsi qu'une extension, d'ailleurs très limitée, en direction de Mortain. Vaste périmètre qui se trouve donc participer de deux régions : Basse-Normandie et Pays de la Loire, et de quatre départements (Orne, Sarthe, Mayenne et, pour une très faible part, Manche).

Entre temps, les Conseils généraux intéressés avaient voté leur participation aux frais d'études; un chargé de mission de la Délégation à l'aménagement du territoire et à l'action régionale pour la mise en place du Parc naturel régional Normandie-Maine prenait bientôt ses fonctions, et s'installait à Carrouges, dans les dépendances du château, futur Centre administratif du Parc; l'étude de reconnaissance, qui constitue la première phase de toute opération de cette nature, pouvait donc commencer. 


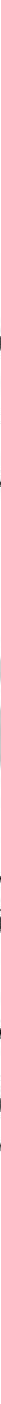

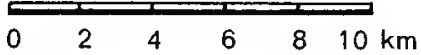

...... Périmetre assigné à l'étude d'aménagement

Routes

Forêt domaniale

Bols partlculiers

Point de vue

$\{$ Arbres et sites remarquables

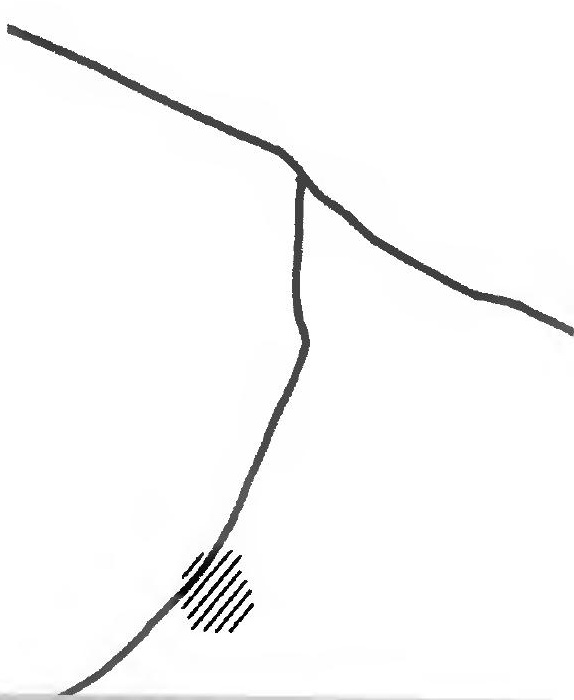




\section{L'ETUDE DE RECONNAISSANCE - LA PARTICIPATION DE L'OFFICE NATIONAL DES FORETS}

L'Office national des forêts, chargé de droit des études pour ce qui est des forêts soumises à sa gestion, et, par affinité, de l'ensemble des études concernant la totalité du patrimoine forestier, sans distinction de propriétaires, se devait de promouvoir une approche réaliste des problèmes posés par la création éventuelle d'un Parc naturel regional, eu égard aux impératifs d'une gestion forestière saine et efficace, dans un secteur éminemment favorable à la production ligneuse, et compte-tenu du rôle déterminant joué par la forêt dans cette affaire. Ce rôle déterminant, la forêt le doit à trois facteurs essentiels :

- d'abord son importance en surface : au total, 45.000 ha, ce qui, pour la zone soumise à reconnaissance, correspond à un taux de boisement de l'ordre de $17 \%$, assez exceptionnel

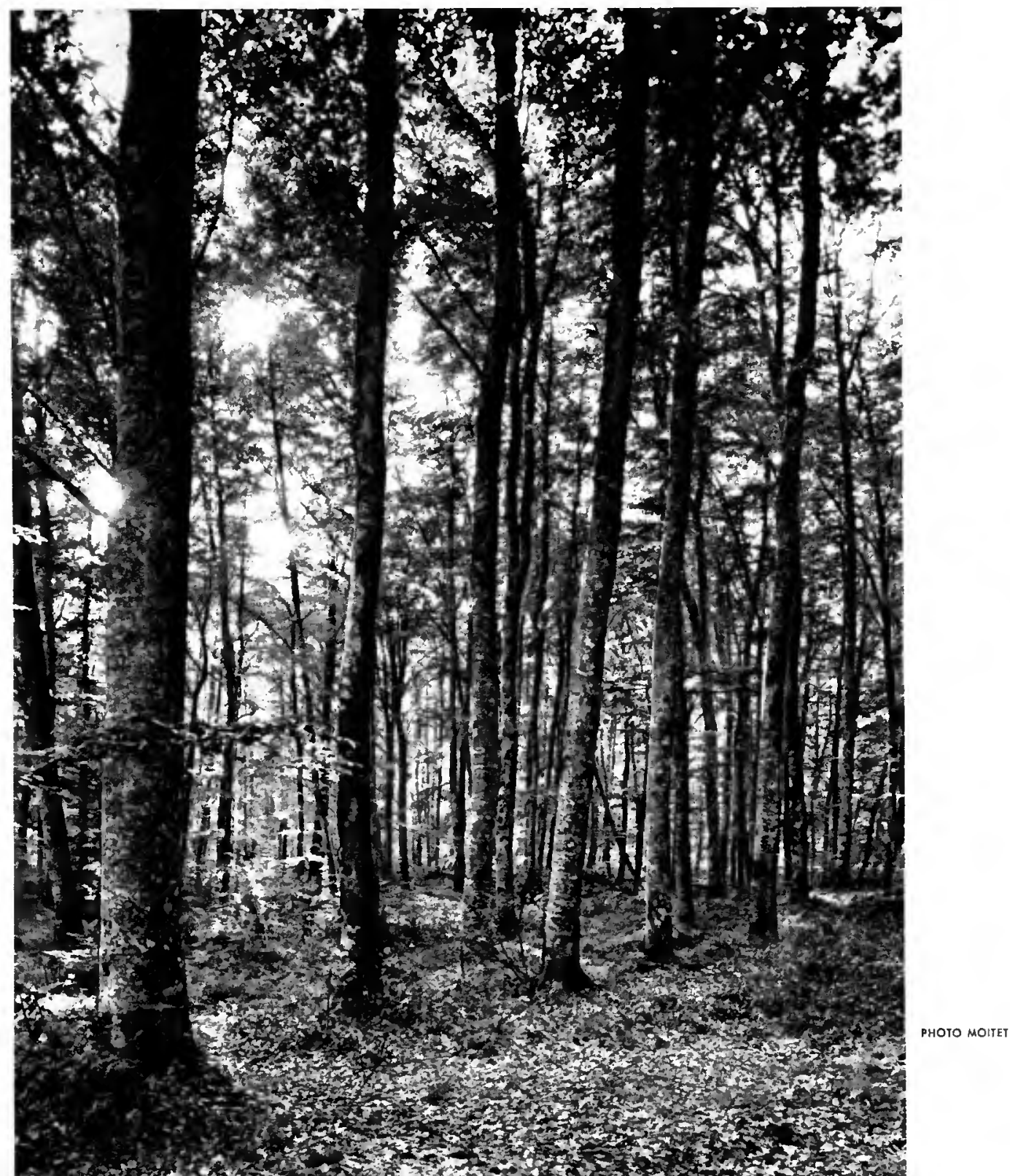


pour les régions de l'Ouest. Sur ce total, 22.000 ha de forêts soumises, toutes domaniales (Ecouves, 8.150 - Andaines, 5.350 - Perseigne, 5.100 - Sillé, 3.400) ;

- ensuite, sa répartition : les massifs dépassant 5.000 ha occupent à eux seuls $56 \%$ de la surface boisée; on passe à près de $70 \%$ pour un seuil fixé à 2.000 ha; l'existence de massifs importants détermine l'effet de masse;

- enfin, sa grande variété, due à un relief relativement accidenté pour la région Ouest (Ecouves culmine à $417 \mathrm{~m}$, comme les Avaloirs) et à une gestion forestière dynamique : forêts en pleine transformation, avec introduction de résineux régulièrement poursuivie sur les sols les plus dégradés, et, ajlleurs, maintien des feuillus; d'où une grande variété d'essences, d'âges et par conséquent de paysages.

L'étude, menée conjointement avec la SEDIAC (Société d'Etudes pour le développement de l'industrie, de l'agriculture et du commerce, d'ailleurs " pilote " de l'étude) est finalement concrétisée par un document d'un peu plus de cent pages, dont une trentaine consacrées aux problèmes forestiers traités en bloc, afin que soient mieux mises en évidence des données par trop méconnues, comme on avait pu le constater lors des nombreuses réunions préparatoires. Ainsi, diverses perspectives avaient été régulièrement évoquées, ici ou là, et en toute bonne foi, allant d'un "classement " pur et simple de la forêt, à sa transformation en un vaste parc zoologique, avec grands animaux visibles en tout lieu et en tout temps. Positions extrêmes, l'une et l'autre incompatibles avec la survie même de ce qu'il s'agit pourtant, au premier chef, de sauvegarder.

Dans ces conditions, la mission du forestier - ce terme étant pris au sens le plus large devient parfaitement claire : s'il ne peut s'opposer à la mise en œuvre d'une politique dictée par les dangers que constituent, pour la survie même de l'homme, une urbanisation et une accélération sans cesse croissantes, il doit par contre poser d'entrée de jeu les limites au-delà desquelles toute entreprise de ce genre irait finalement à l'encontre du but poursuivi.

Le rapport d'étude de reconnaissance met l'accent sur les points les plus importants à cet égard :

a) la forêt est un domaine vivant; l'arbre vit et meurt;

b) la forêt, richesse économique importante, sollicitée à l'aval par des besoins et des industries en perpétuelle évolution, doit s'adapter aux exigences de son temps.

Le grand public doit dès lors admettre que se transforment sous ses yeux des paysages auxquels il s'accoutume trop bien, et comprendre que substitutions d'essences, rénovations, régénérations ne signifient nullement massacre. II doit aussi comprendre qu'en dernière analyse, la forêt ne peut concourir à une saine politique de l'environnement, si elle ne se fonde sur une production ligneuse économiquement intéressante, seule susceptible de permettre son renouvellement, donc sa pérennité, et un minimum d'éntretien ;

c) la forêt, facteur de l'équilibre biologique général, ne subsiste elle-même que dans le respect d'équilibres internes fondamentaux; à preuve le nécessaire équilibre sylvo-cynégétique ;

d) enfin, la forêt est menacée par l'homme lui-même, trop souvent mal informé ; ainsi la forêt doit être préservée du feu; ainsi la forêt doit rester propre; bref, une fréquentation touristique accrue ne peut se concevoir dans l'anarchie, ni sans un minimum de contraintes. Tels sont les principaux thèmes développés, qui constituent sans doute autant d'évidences pour un forestier averti, mais qui ne manquent pas toujours de nouveauté pour le profane.

Finalement, le rapport d'étude de reconnaissance devait être éxaminé par la Commission interdépartementale chargée de suivre les travaux (Commission composée des autorités préfectorales et des représentants des grands services et organismes socio-professionnels concernés, aux échelons régionaux et départementaux). Lors de sa réunion du 2 février 1970, les décisions suivantes furent prises :

- poursuite de l'opération, c'est-à-dire étude d'aménagement et établissement d'avantprojets d'exécution, confiés également à la SEDIAC et à l'Office national des forêts.

- modification du périmètre : au total, environ 135.000 ha, comprenant 130 communes (82 dans l'Orne, 24 dans la Mayenne, 20 dans la Sarthe, 4 dans la Manche) au lieu de 260.000 ha primitifs, mais les massifs forestiers importants demeurent concernés.

- création de commissions locales d'information, au nombre de neuf, qui se partagent la zone retenue, et comprennent chacune des élus locaux, et des représentants d'organismes socio-professionnels ou autres (tels les Syndicats de propriétaires forestiers sylviculteurs - 
Tous ces éléments doivent, en dernière analyse, servir à élaborer le projet de charte constitutive, indispensable pour que puisse intervenir le décret de classement définitif.

C'est dans ce cadre général que l'Office national des forêts a pour mission particulière, au stade actuel :

- de définir par grande forêt domaniale les aménagements souhaitables;

- de définir et localiser un observatoire de la "forêt vivante ";

- de préciser, sur proposition conjointe des Centres régionaux de la propriété forestière et des Syndicats de propriétaires forestiers sylviculteurs, la place de la forêt privée dans l'aménagement du Parc;

- enfin, d'étudier les dispositions et conventions qui permettront une juste conciliation entre l'aménagement du Parc naturel régional et le maintien d'une économie forestiére productive, l'étude de reconnaissance ayant bien mis l'accent sur ce point.

Ces divers aspects du problème sont explicités ci-après, s'agissant d'ailleurs de simples réflexions préparatoires, qui engagent seulement l'auteur du présent article; elles ne préjugent pas des résultats définitifs de l'étude en cours, ni des conclusions qui en seront tirées.

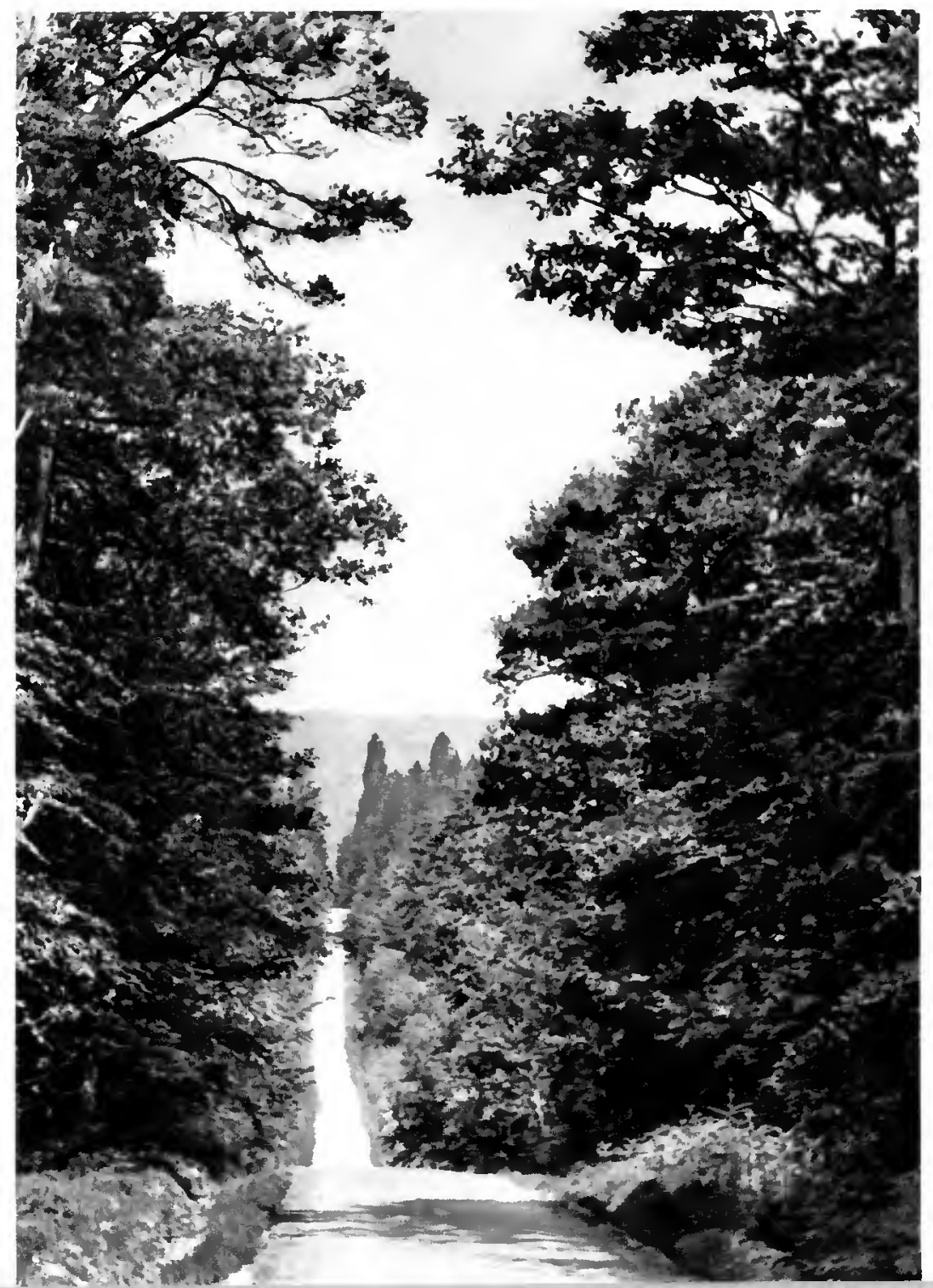




\section{Les aménagements souhaitables en forêt domaniale}

II s'agira d'abord de poursuivre une politique d'ores et déjà largement amorcée :

- le réseau de routes forestières a déjà été grandement amélioré, par financements conjoints de l'Etat et des départements de l'Orne et de la Sarthe, chacun en ce qui le concerne; ce réseau peut être considéré comme suffisant, à de rares exceptions près;

- des sentiers pédestres, des itinéraires équestres ont d'ores et déjà été définis et baljsés; mais il faudra les compléter, et parfois les intléchir, afin qu'ils se raccordent avec les itinéraires équestres hors forêt ou avec les sentiers de grande randonnée, organisés au plan national ;

- de même, en matière de menus équipements d'accueil - tels les aires, voire les abris pour pique-nique - il suffira de compléter les installations existantes.

Mais il faudra aussi, et sans que cette énumération soit exhaustive, porter attention à des problèmes relativement nouveaux, savoir :

- problème des relais pour randonneurs équestres et pédestres : une opération pilote est en cours en forêt domaniale d'Ecouves sous forme d'aménagement d'une maison forestière désaftectée.

- problème des campings : il serait raisonnable d'aménager un camping de bon standing dans chaque massif, si possible en périmètre, et à proximité d'une localité disposant d'un minimum d'intrastructure commerciale.

- problème des parkings : ce point mérite une recherche particulière, et sans doute une orientation vers le parking diffus (plutôt que terre-pleins dénudés, inesthétiques, qui transposeraient en forêt un élément du monde urbain).

- problème d'un éventuel parc animalier; reste à trouver une localisation judicieuse, après avoir opté entre les deux formules possibles : aménagement à caractère zoo (plusieurs enceintes de faible surface, reliées par des sentiers de promenade, et permettant au visiteur de voir des animaux depuis l'extérieur, sans aucune difficulté), ou parc animalier véritable, nécessitant alors une grande surface, avec infrastructure de pénétration, et toutes les sujétions techniques et surtout financières qu'une telle formule comporte.

- problème des zones de silence : il est à peine besoin d'insister sur leur intérêt; il devrait s'agir de surtaces de plusieurs centaines d'hectares, dans lesquelles toute circulation automobile serait interdite, sauf pour les ayants-droits de la forêt.

\section{L'observatoire de la forêt vivante}

II s'agit tout simplement de déterminer, dans un ou deux massifs, des itinéraires de promenade judicieusement renseignés, de telle sorte que le visiteur puisse, en un temps limité, faire connaissance avec le plus grand nombre possible d'essences, de peuplements, de techniques, propres à susciter sa réllexion sur les problèmes forestiers.

\section{La place de la forêt privée dans l'aménagement du parc}

II conviendra de transcrire les suggestions et avis des syndicats ou organismes compétents en la matière. En tout état de cause, il semble bien que toute participation directe d'une torêt particulière à l'aménagement du Parc ne saurait être que volontaire, suivant baux ou conventions librement consentis, et précisant dans chaque cas les conditions financières. La charte n'en devra pas moins résoudre les graves problèmes posés, sur un plan général, par l'accroissement de la fréquentation touristique probable partout, même en dehors de toute action spécitique; notamment aggravation des risques d'incendie et de déprédations diverses. Des formules originales doivent être recherchées, susceptibles de donner toutes les garanties nécessaires. De même en matière d'exercice du droit de chasse, ceci valant également pour les forêts domaniales.

Les dispositions et conventions qui permettront une juste conciliation entre l'aménagement du Parc ef le maintien d'une économie forestière productive.

C'est un point essentiel.

La création de séries touristiques, où l'objectif production ligneuse est délibérément considéré comme secondaire (telle, en forêt domaniale des Andaines, et à proximité immédiate de Bagnoles de l'Orne, une série d'environ 130 ha dont l'aménagement sera entrepris suivant un 
plan en cours d'étude); la rénovation très progressive de certains sites; la désignation d'arbres remarquables - ou susceptibles de le devenir - à conserver lors des passages en coupe, même au défi des règles sylvicoles; le maintien, dans les forêts en transformation, de séries feuillues plus importantes qu'elles ne devraient l'être si l'on s'en tenait strictement aux seules considérations économiques; la conservation, lors des enrésinements, en bordure des voies fréquentées, de bandes intactes - ou la plantation de quelques lignes feuillues, voire ornementales - autant de mesures parfaitement justifiées.

Mais on ne saurait aller plus loin sans compromettre la production ligneuse.

La charte du Parc devra bien mettre l'accent sur le fait que cette production, au demeurant créatrice d'emplois et source de vie économique appréciable sur le plan local, constitue un objectif prioritaire. Elle devra prévoir toutes mesures utiles pour que les activités qui lui sont liées, telles celles exercées par les exploitants forestiers, ne soient nullement entravées, pour que la forêt elle-même ne soit pas menacée, et pour que ses nécessaires transformations ne soient pas remises en cause.

\section{En concluslon : forêt de production, ou forêt-loisirs ?}

Dans une région comme la Normandie, particulièrement favorable à la croissance de l'arbre, en même temps que située à proximité relative de l'agglomération parisienne, un choix est-il possible?

Parallèlement, la forêt peut-elle rester productive, tout en permettant la récréation de l'homme? Est-ce une gageure?

D'aucuns le pensent. Faut-il donc se replier et s'isoler à l'intérieur des périmètres ? Serait-ce efficace à terme, dès l'instant où un projet du type Parc naturel régional existe, quí nous intéresse directement tout en débordant largement nos "frontières "? N'appartient-il pas plutôt au forestier d'être présent, afin de marquer, au jour le jour, les limites d'une coexlstence acceptable entre les deux aspects de la forêt de notre temps, et de mettre à profit, pour une meilleure et nécessaire information du public, le surcroît d'intérêt qu'elle suscite? Au cas particulier, c'est la politique suivie par l'Office national des forêts. Puisse-t-elle porter ses fruits!.. 